\title{
Occurrence of Unreduced Female Gametes Leads to Sexual Polyploidization in Lantana
}

\author{
David M. Czarnecki II and Zhanao Deng ${ }^{1}$ \\ University of Florida, IFAS, Environmental Horticulture, Gulf Coast Research and Education Center, \\ 14625 C.R. 672, Wimauma, FL 33598
}

AdDitional INDEX words. polyploid, ploidy manipulation, ploidy analysis, $2 n$ gametes, invasiveness control

\begin{abstract}
Lantana camara, a member of the verbena family, is a popular ornamental yet highly invasive plant. It can escape from cultivation through seed dispersal and can contaminate native lantana species (Lantana depressa) through cross-pollination. Ploidy manipulation is being used as a genetic approach to produce sterile, noninvasive lantana cultivars. Polyploids have been observed in lantana (Lantana), but little information is available about the mechanisms for lantana polyploidization and the possible effects of natural polyploidization on the sterility (or fertility) of lantana triploids. In this study, we analyzed the ploidy level of more than 1500 lantana progeny from self, open, and/or controlled pollinations of $\mathbf{1 0}$ commercial cultivars and seven breeding lines. Our results confirmed the occurrence of unreduced gametes, specifically, unreduced female gametes (UFGs), in lantana. The frequency of UFG formation varied among commercial cultivars, and cultivars/breeding lines could be categorized into two groups: UFG producers and nonproducers. Tetraploid cultivars Gold, Pink Caprice, and Radiation fall into the UFGproducing group, while diploid cultivars Cream, Denholm White and Lola and tetraploid cultivars Carlos, Dallas Red and Irene belong to the nonproducer group. The frequency of UFG formation observed in nine UFG producers was $5.5 \%$ to $100 \%$, varying with cultivar, growing condition, and/or pollination scheme. Progeny of the cross between 'Carlos' (seed parent) and 'Gold' (pollen parent) also showed the ability to produce UFGs, indicating that the trait (UFG formation) could be transmitted from 'Gold' to its progeny and is likely to be controlled by nuclear gene(s). Lantana triploids with or without the UFG-forming ability in its genetic background showed a significant difference in seed set: the former produced abundant seed when pollinated, while the latter produced little or no seed. The results stress the need to avoid using lantana with UFG-forming ability as parents in crosses designed to produce sterile triploids for invasiveness control. Additionally, the results from this study suggest multiple pathways for emergence and evolution of polyploids in cultivated and naturalized lantana populations.
\end{abstract}

Lantana is a member of the Verbenaceae and has been widely grown as a container plant, hanging basket plant, groundcover, hedge, or accent plant (Howard, 1969). It is popular in the nursery industry because of easy propagation and short production cycles, and is popular among gardeners because it attracts butterflies and is tolerant to droughts and poor soil conditions. Many nursery growers produce lantana, especially in the southern United States. For example, a survey of the Florida nursery industry, which consists of more than 5000 nurseries, indicates that $19 \%$ of the responding nurseries grew lantana and that the annual sales value in Florida alone was over $\$ 40$ million (Wirth et al., 2004). The majority of the commercial lantana cultivars belong to Lantana camara. This species can escape from cultivation through seed dispersal and can invade agricultural and natural lands and hybridize with native lantana species (Lantana depressa). Because of these behaviors, L. camara has been listed as an invasive species in southern and central Florida (Florida Exotic Pest Plant Council, 2007). In several other countries including Australia, India, and South Africa, lantana is considered as a noxious weed or an

Received for publication 14 Aug. 2009. Accepted for publication 28 Sept. 2009. This project was funded in part by the Florida Nursery Growers and Landscape Association, the Southwest Florida Water Management District, and the USDA/CSREES/TSTAR (United States Department of Agriculture/Cooperative States Research, Extension and Education Service/Tropical and Subtropical Agriculture) program.

We thank Joyce Jones, Gail Bowman, and Sarah Smith for their technical assistance, and Dr. Jude W. Grosser, J.L. Chandler, and Patricia Brickman for ploidy analysis of lantana commercial cultivars in 2004.

${ }^{1}$ Corresponding author. E-mail: zdeng@ufl.edu. invasive species (Sharma et al., 2005). Polyploid manipulation, particularly triploid production, has been proposed as a genetic approach to develop sterile, noninvasive lantana cultivars (Czarnecki et al., 2008). Similar genetic approaches (polyploid production and selection) are being used to sterilize other ornamental plants for invasiveness control (Ranney, 2004).

Polyploids are common in L. camara. Triploids, tetraploids, pentaploids, and hexaploids have been reported in cultivated and naturalized L. camara (Czarnecki et al., 2008; Natarajan and Ahuja, 1957; Spies and Stirton, 1982). Several lines of evidence suggest that polyploidization may be associated with the species' invasive behavior (Sanders, 2001): Tetraploids are rare in the native populations of this species in tropical America, but are very common in the naturalized populations in Australia, India, and South Africa. Overall, tetraploids have courser leaves, grow more vigorously, and set more seeds (Sanders, 2001), whereas diploids tend to be dwarf and stunted. Tetraploids have a much wider range of distribution than diploids, and pentaploids are frequently found at high altitudes (Sinha and Sharma, 1984).

In plants, natural polyploidization can occur through somatic chromosome doubling or gametic nonreduction (Bretagnolle and Thompson, 1995). The former results from mitotic abnormalities in somatic (zygotic and meristematic) cells, while the latter results from meiotic abnormalities during gamete or gametophyte genesis and the formation of unreduced gametes (pollen and/or eggs). When an unreduced gamete unites with another unreduced gamete (bilateral) or with a normal haploid gamete (unilateral), the union leads to sexual polyploidization. To a certain extent, polyploidization via somatic chromosome 
doubling bears similarity to inbreeding, while polyploidization via unreduced gametes can retain heterozygosity. Thus, the two polyploidization processes can have significant differences in terms of genetic and evolutionary consequences to polyploidized species (Bretagnolle and Thompson, 1995; Hermsen, 1984).

Little information is available regarding the origin of polyploids in L. camara, except for a report by Khoshoo and Mahal (1967). The authors observed several tetraploids and a pentaploid in the open-pollinated (OP) progeny of a triploid and two hexaploids in the OP progeny of a pentaploid. They inferred that these tetraploid and hexaploid progeny with chromosome numbers higher than their parents must have come from union of unreduced female gametes with normally reduced pollen. The occurrence of UFGs has been reported in several plant species (Ramsey and Schemske, 1998; Stelly and Peloquin, 1986). Unreduced pollen has been reported in many plants and seems to be a more common mode of sexual polyploidization in plants (Bretagnolle and Thompson, 1995). Several studies have examined the pollen size and morphology of Lantana, but none of them reported the occurrence of unreduced pollen in $L$. camara (Raghavan and Arora, 1960; Sanders, 1987). In a recent pollen viability study, we examined tens of thousands of pollen grains and did not notice highly variable viable pollen grains within cultivars (D. Czarnecki and Z. Deng, unpublished).

During the course of interploid pollination and triploid generation, we observed pentaploid progeny from a tetraploid by diploid cross, which seems to indicate the occurrence of UFGs in the lantana cultivars used. Therefore, a study was undertaken to confirm the occurrence of UFGs in lantana, to determine its frequency and distribution in major commercial lantana cultivars, to test its transmissibility from generation to generation, and to determine if this trait would affect seed set on lantana triploids. Toward these objectives, progeny of commercial cultivars from self-pollination (SP) and OP were first analyzed for ploidy levels, followed by controlled pollinations among cultivars and breeding lines and ploidy analysis of their progeny. This report presents the results from these pollinations and ploidy analyses. Based on the results, we further propose a model for the origin of the multiple levels of polyploids in cultivated (and naturalized) L. camara and discuss the possible mechanisms for the formation of unreduced gametes in lantana and potential implications of this trait for lantana ploidy manipulation, particularly triploid generation and selection for sterile lantana development.

\section{Materials and Methods}

Plant materials. Ten commercial cultivars and seven breeding lines were used in this study (Table 1). Cuttings or rooted cuttings of these cultivars were generously provided by B. Dehgan (University of Florida, Gainesville) in 2004, and Robrick Nursery (Hawthorne, FL) in 2005 and 2006. Plants were grown in plastic containers filled with a commercial soilless mix, VerGro container mix A (Verlite, Tampa, FL) amended with controlled-release fertilizer, Osmocote $(15 \mathrm{~N}-$ 3.9P-10K, 8 to 9 months release at $21{ }^{\circ} \mathrm{C}$; Scotts, Marysville, $\mathrm{OH})$ at $7.12 \mathrm{~kg} \cdot \mathrm{m}^{-3}$.

Three types of pollination were completed to generate as many seeds (progeny) as possible for ploidy analysis: SP, OP, and controlled pollinations (CP). In SP, lantana plants in 11.4-L containers were grown in a greenhouse with double doors and a thrips-proof screen. Insecticides were applied at regular intervals in the greenhouse. As lantana is autogamous (Rambuda and Johnson, 2004), hand pollination is not needed for SP. Therefore, to avoid damages to flowers and maximize seed set, flowers were allowed to self-pollinate without manual emasculation or hand pollination. In OP, lantana plants in $26.5-\mathrm{L}$ containers were arranged on a raised metal bench outside in open air. Different cultivars were interspersed to maximize

Table 1. Ploidy level, ancestry, and source of Lantana camara cultivars and breeding lines used, and their ability and frequency to produce $2 n$ female gametes observed in this study.

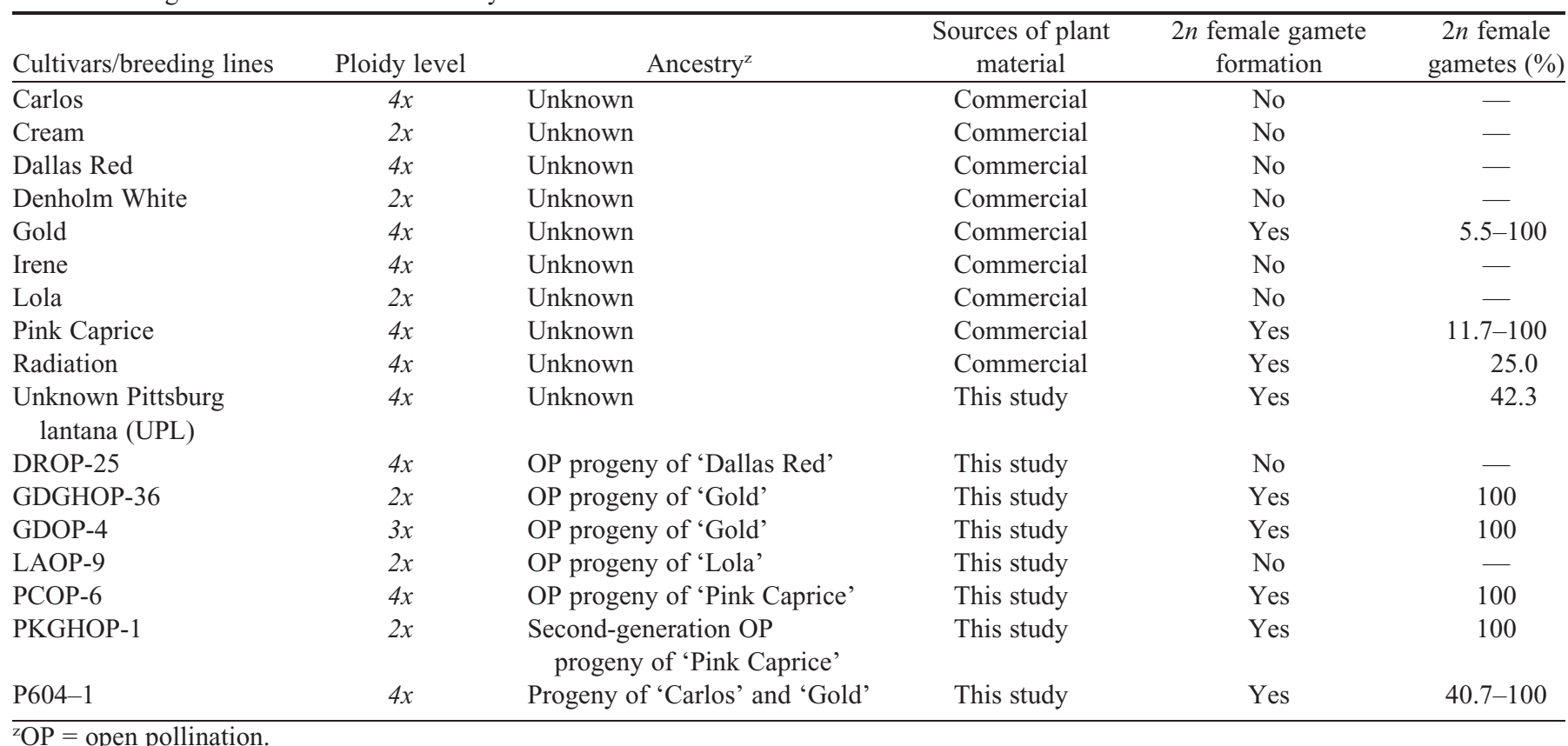


cross-pollination mediated by insect pollinators. Insecticides were not applied. Common insect pollinators observed were butterflies, moths, bees, and wasps. CP were done on lantana plants grown in the greenhouse. Mature flowers were emasculated by pulling off the entire calyx to which the anthers were attached and pollinated immediately using a camel brush with fresh pollen from flowers that just opened. Between pollinations, the brushes were soaked in $100 \%$ ethanol to kill residual pollen. Controlled pollinations were done from Apr. to June 2006, Aug. to Dec. 2006, Feb. to July 2007, June to Nov. 2008, and Feb. 2009.

When lantana fruit (berries) turned dark purple to black and became ripe, they were collected from SP, OP, or CP flower heads. Seeds were extracted, cleaned, and sown within 1 to 2 weeks after harvest, with the exception of the SP seeds of 'Pink Caprice', which were cleaned and stored for over 1.5 years before sowing.

Progeny Growing. Seeds were sown on the surface of a peat/vermiculite mix (VerGro container mix A) and germinated under intermittent mist in a greenhouse. Temperatures in the greenhouse ranged from $16^{\circ} \mathrm{C}$ (night) to $30^{\circ} \mathrm{C}$ (day), and no artificial lighting was used. The majority of seeds germinated within 4 months after sowing, but some took as long as 1 year to germinate. After young seedlings had developed true leaves, they were transferred to $12.7-\mathrm{cm}$-diameter plastic containers filled with VerGro container mix A. Plants were fertilized by incorporating Osmocote $(15 \mathrm{~N}-3.9 \mathrm{P}-10 \mathrm{~K})$ at $7.12 \mathrm{~kg} \cdot \mathrm{m}^{-3}$ in the soilless mix.

Ploidy analysis. Analysis was performed on all plants using fully expanded young leaves and the Partec PA I ploidy analyzer and the CyStain ultraviolet Ploidy Precise $\mathrm{P}$ dye (Partec, Münster, Germany). The manufacturer-recommended ploidy analysis procedure was followed with minor modifications. The ploidy level of cultivars and progeny was determined by comparing to one or more commercial cultivars (reference cultivars) with known ploidy levels that were included in the same analysis.

The ploidy levels of the reference cultivars had been confirmed by counting chromosomes in root tip cells. Growing root tips of 'Cream', 'Gold', 'Pink Caprice', and 'Radiation' were collected from rooted cuttings, chilled at $8{ }^{\circ} \mathrm{C}$ overnight, and pretreated with $0.05 \%$ colchicine at ambient temperature for $4 \mathrm{~h}$, fixed in Carnoy's fluid for $2 \mathrm{~d}$, and stored in $70 \%$ ethanol at $4{ }^{\circ} \mathrm{C}$. Fixed root tips were hydrolyzed in $1 \mathrm{~N} \mathrm{HCl}$ at $60^{\circ} \mathrm{C}$ for 5 to $10 \mathrm{~min}$, squashed in acetic carmine on glass slides, and observed under a $100 \times$ objective on a microscope (BH-2; Olympus, Tokyo). Chromosome counting results showed that 'Cream' is a diploid $(2 n=2 x=22)$, and 'Gold', 'Pink Caprice', and 'Radiation' are tetraploids $(2 n=2 x=44)$.

\section{Results}

Ploidy analysis of SP Progeny. One diploid ('Lola') and two tetraploid cultivars ('Pink Caprice' and 'Gold') were allowed to self-pollinate naturally in the greenhouse (without emasculation and hand-pollination). More than 900 seeds were collected and sown, and more than 500 progeny were analyzed for ploidy levels. All 103 progeny of 'Lola' were diploids, indicating no UFG formation in this cultivar. The majority $(88.3 \%$ and $92.3 \%)$ of the progeny of tetraploid cultivars Pink Caprice and Gold were tetraploids, as expected for normal $n$ (2x) gamete formation and fertilization (Table 2), but 5.5\% of 'Gold' SP progeny and $11.7 \%$ of 'Pink Caprice' SP progeny were hexaploids (Table 3 ). The occurrence of these hexaploids indicates that a $2 n$ gamete $(4 x)$ had been formed, combined with an $n$ gamete $(2 x)$, and developed into a hexaploid embryo. Additionally, two diploids were recovered from 'Gold' SP seeds, but no diploids were identified in the SP progeny of 'Pink Caprice', even though much more progeny (311 of 'Pink Caprice' vs. 91 of 'Gold') were examined. This is the first time that such ploidy level reduction from a tetraploid to a diploid has been observed in lantana. Understanding the origin of these diploids will require the use of cytological and molecular genetic analysis tools, which are not yet available in lantana.

The above results clearly indicate the formation of $2 n$ gametes in 'Gold' and 'Pink Caprice', but do not elucidate which side (maternal or paternal) produced the $2 n$ gametes.

Ploidy analysis of OP Progeny. To confirm the above findings and to determine if $2 n$ gametes occur in other lantana cultivars, OP seeds (more than 2800 in total) were collected from 'Lola', 'Pink Caprice', and 'Gold', as well as another two diploids ('Cream' and 'Denholm White') and three more tetraploids ('Carlos', 'Dallas Red', and 'Irene') grown in a large screen house. If $2 n$ gametes were not present, it would be expected that most OP progeny of a diploid cultivar would be diploid (from SP or CP by other diploid cultivars) and some progeny would be triploid (from $\mathrm{CP}$ by tetraploid cultivars) (Table 2). Similarly, if $2 n$ gametes did not occur, most OP progeny of a tetraploid cultivar would be tetraploids (from SP or CP by other tetraploid cultivars) and some progeny would be triploids (from CP by diploid cultivars) (Table 2). On the other hand, should $2 n$ gametes have been formed on the seed parent side, some OP progeny of a diploid cultivar would be expected to be tetraploids (from $2 n$ gametes of the diploid seed parent and the normal gametes of tetraploid pollen parents), and some OP progeny of a tetraploid cultivar would be pentaploids and hexaploids (from $2 n$ gametes of the tetraploid seed parent and the normal $n$ gametes of diploid and tetraploid pollen parents) (Table 2).

Table 2. Expected ploidy levels in the progeny of diploid and tetraploid lantana with or without formation of $2 n$ female and/or male gametes. Expected ploidy levels in progeny of diploid and tetraploid parents with and/or without $2 n$ gamete formation during sporogenesis

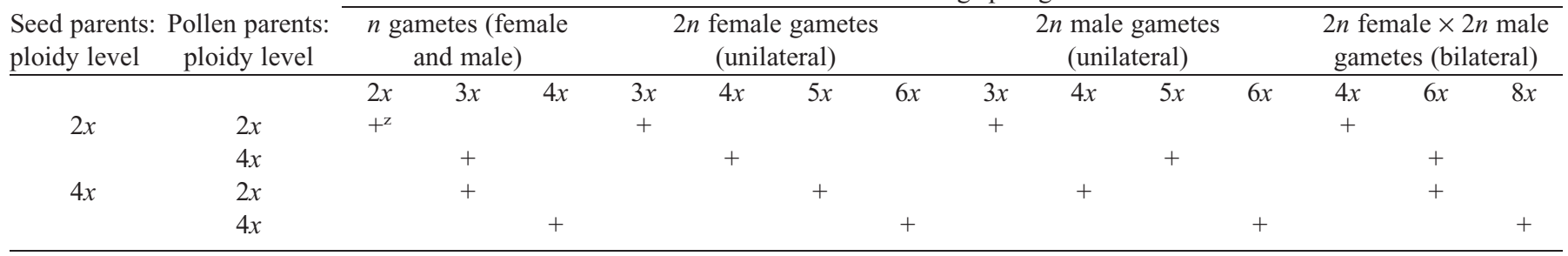

z“+" indicates a likely appearance of such ploidy level in the progeny. 
A limited number of seeds (and progeny) were obtained from 'Cream' and 'Denholm White' due to their very low female fertility, especially in 'Denholm White', where 2472 flowers were pollinated from five crosses and $0.04 \%$ seed set (one seed) was observed. All their progeny were diploids, indicating a possible lack of $2 n$ gamete formation in these cultivars (more supporting data below).

The majority (96.4\%) of 'Lola' OP progeny were diploids and 2 of 55 progeny were triploids (Table 3). In an OP environment and with diploids and tetraploids present, these two triploids could have resulted from normal gamete formation and fertilization, or $2 n$ gamete formation and fertilization (Table 2). To determine the origin of the two triploids, we examined the leaf and flower morphology of these triploids, as well as all the potential diploid and tetraploid parents present at the time when OP was conducted. The most obvious difference was in flower color: all diploids had yellow, creamy yellow, or white flowers, while all tetraploids had pink, magenta, or red flowers. Our lantana flower color inheritance studies indicated that crosses among yellow, creamy yellow, or white flowers would result in progeny with shades of yellow to white, and crosses between yellow, creamy yellow, or white flowers and pink, magenta, or red flowers would result in progeny with shades of pink or red (D. Czarnecki and Z. Deng, unpublished). Flowers of the two OP triploids of 'Lola' were in shades of magenta, indicating a possible origin from union of normal $n(x)$ female gametes of 'Lola' (yellow) with $n(2 x)$ male gametes of tetraploids (pink or magenta). Thus, $2 n$ gametes were probably not involved in the formation of the triploid progeny of 'Lola'.

Four ploidy levels $(3 x, 4 x, 5 x$, and $6 x)$ were observed in 14 'Pink Caprice' OP progeny (Table 3). The presence of $5 x$ and $6 x$ progeny indicates the occurrence of $2 n$ gametes in 'Pink Caprice' (Table 2). Further, the presence of $5 x$ suggests that, more specifically, UFGs occurred in 'Pink Caprice' (Table 2). The percentage of $5 x$ and $6 x$ progeny was $35.7 \%$, a much higher frequency of $2 n$ gamete formation than observed when 'Pink Caprice' was grown in the greenhouse and self-pollinated. Formation of $2 n$ gametes was observed again in 'Gold', as indicated by the presence of 11 pentaploids and 4 hexaploids in the 81 'Gold' OP progeny (Table 3). Similarly, the frequency of $2 n$ gamete formation in 'Gold' was much higher in the OP progeny than in the SP progeny $(14.3 \%$ vs. $5.5 \%$ in the SP progeny). One diploid appeared in 'Gold' OP progeny, and its causes remain to be understood.

In 'Carlos' and 'Dallas Red', the majority of the 77 or 50 OP progeny were tetraploids and a few were triploids. The absence of pentaploids or hexaploids indicates a lack of $2 n$ gamete formation in these tetraploid cultivars.

OP seeds were also collected from a commercial cultivar grown at a hotel site in Pittsburgh, PA. This unknown Pittsburgh lantana (UPL) and its progeny were tetraploid or hexaploid, suggesting a high frequency (57.7\%) of $2 n$ gamete formation in this cultivar (Table 3).

These results from ploidy analysis of OP progeny confirm the results from SP progeny analysis. They also suggest considerable differences among lantana cultivars in $2 n$ gamete formation and the nonreduction is likely to occur on the maternal side.

Ploidy analysis of CP Progeny. A total of over 2000 seeds were sown from CP. 'Carlos' $(4 x)$ produced tetraploid progeny when pollinated with 'Dallas Red' $(4 x)$ and produced triploids (214) when pollinated with 'Denholm White' $(2 x)$, 'Lola' $(2 x)$, or a diploid breeding line LAOP-9 (progeny of 'Lola') (Table 4). These results support the above-mentioned observation in SP and OP progeny: 'Carlos' did not produce $2 n$ gametes. It seems reasonable to make a similar conclusion for 'Dallas Red', as it produced only tetraploid progeny when pollinated with 'Carlos' and produced only triploids when pollinated with 'Denholm White', 'Lola', or LAOP-9 (Table 4). When pollinated with three tetraploids ('Carlos', 'Dallas Red', and 'Irene'), 'Lola' produced triploids, and when pollinated with diploid LAOP-9 or selfed, 'Lola' produced diploids (Table 4). 'Cream' produced triploids when pollinated with 'Carlos', 'Dallas Red', or 'Irene', which indicates that 'Cream' did not form $2 n$ gametes and supports the observations from SP and OP progeny (Table 4).

Although 1137 flowers of 'Gold' were pollinated with 'Carlos' or 'Dallas Red' in 2007 and 2008, only 20 seeds were obtained and only 15 germinated for ploidy analysis. The high

Table 3. Distribution of ploidy levels in lantana progeny from self and open pollination (SP and OP). Pollination was performed in 2006 and 2007, and ploidy analysis was done in 2007 and 2008 at Wimauma, FL.

\begin{tabular}{|c|c|c|c|c|c|c|c|c|c|}
\hline \multirow{2}{*}{$\begin{array}{l}\text { Seed parent } \\
\text { (ploidy level) }\end{array}$} & \multirow{2}{*}{$\begin{array}{l}\text { Pollination } \\
\text { scheme }^{z}\end{array}$} & \multirow{2}{*}{$\begin{array}{l}\text { Seeds collected } \\
\text { and sown (no.) }\end{array}$} & \multirow{2}{*}{$\begin{array}{c}\text { Progeny available for } \\
\text { ploidy analysis (no.) }\end{array}$} & \multicolumn{5}{|c|}{ Progeny in ploidy levels (no.) } & \multirow{2}{*}{$\begin{array}{c}2 n \text { gametes } \\
(\%)\end{array}$} \\
\hline & & & & $2 x$ & $3 x$ & $4 x$ & $5 x$ & $6 x$ & \\
\hline 'Lola' $(2 x)$ & SP & 263 & 103 & 103 & & & & & 0 \\
\hline 'Pink Caprice' $(4 x)$ & SP & 403 & 298 & & & 263 & & 35 & 11.7 \\
\hline 'Carlos' $(4 x)$ & SP & 38 & 15 & & & 15 & & & 0 \\
\hline 'Cream' $(2 x)$ & OP & 56 & 9 & 9 & & & & & 0 \\
\hline 'Pink Caprice' $(4 x)$ & OP & 403 & 14 & & 2 & 7 & 4 & 1 & 35.7 \\
\hline 'Gold' $(4 x)$ & OP & 758 & 61 & 1 & 1 & 52 & 6 & 3 & 14.3 \\
\hline 'Carlos' $(4 x)$ & OP & 639 & 77 & & 9 & 68 & & & 0 \\
\hline 'Dallas Red' (4x) & OP & 254 & 50 & & 3 & 47 & & & 0 \\
\hline 'Irene' $(4 x)$ & OP & 355 & 52 & & 1 & 51 & & & 0 \\
\hline $\operatorname{UPL}^{\mathrm{x}}(4 x)$ & OP & 65 & 26 & & & 11 & & 15 & 57.7 \\
\hline
\end{tabular}

zTwo pollination schemes were used. In SP, flowers were allowed to self-pollinate without manual emasculation and hand pollination in a greenhouse. In OP, lantana plants were placed outside in open air, and different cultivars were interspersed to maximize cross-pollination mediated by insect pollinators.

${ }^{y}$ Numbers enclosed in squares are the numbers of polyploid progeny indicating $2 n$ gamete formation.

${ }^{\mathrm{x} U n k n o w n}$ Pittsburgh lantana. 
Table 4. Distribution of ploidy levels in lantana progeny of controlled pollinations grouped by seed parent. Pollinations were made in 2005, 2006, and 2007, and ploidy analysis done in 2008 at Wimauma, FL.

\begin{tabular}{|c|c|c|c|c|c|c|c|c|c|}
\hline \multirow[b]{2}{*}{ Seed parent } & \multirow[b]{2}{*}{ Pollen parent } & \multirow{2}{*}{$\begin{array}{c}\text { Flowers } \\
\text { pollinated (no.) }\end{array}$} & \multirow{2}{*}{$\begin{array}{l}\text { Progeny available for } \\
\text { ploidy analysis (no.) }\end{array}$} & \multicolumn{5}{|c|}{ Progeny in ploidy levels (no.) } & \multirow{2}{*}{$\begin{array}{c}2 n \text { gametes } \\
(\%)\end{array}$} \\
\hline & & & & $2 x$ & $3 x$ & $4 x$ & $5 x$ & $6 x$ & \\
\hline 'Carlos' (4x) & 'Dallas Red' (4x) & 2202 & 31 & & & 31 & & & 0 \\
\hline 'Carlos’ $(4 x)$ & 'Denholm White' $(2 x)$ & 731 & 23 & & 23 & & & & 0 \\
\hline 'Carlos' $(4 x)$ & LAOP-9 $(2 x)$ & 1259 & 56 & & 56 & & & & 0 \\
\hline 'Carlos' $(4 x)$ & 'Lola' $(2 x)$ & 3656 & 135 & & 135 & & & & 0 \\
\hline 'Dallas Red' (4x) & 'Carlos' $(4 x)$ & 2051 & 19 & & & 19 & & & 0 \\
\hline 'Dallas Red' (4x) & 'Denholm White' $(2 x)$ & 468 & 5 & & 5 & & & & 0 \\
\hline 'Dallas Red' (4x) & LAOP-9 $(2 x)$ & 1653 & 28 & & 28 & & & & 0 \\
\hline 'Dallas Red' (4x) & 'Lola' $(2 x)$ & 1839 & 1 & & 1 & & & & 0 \\
\hline 'Lola' $(2 x)$ & LAOP-9 $(2 x)$ & 1894 & 100 & 100 & & & & & 0 \\
\hline 'Lola' $(2 x)$ & 'Lola' $(2 x)$ & 567 & 18 & 18 & & & & & 0 \\
\hline 'Lola' $(2 x)$ & 'Carlos' $(4 x)$ & 2215 & 16 & & 16 & & & & 0 \\
\hline 'Lola' $(2 x)$ & 'Dallas Red' (4x) & 2230 & 5 & & 5 & & & & 0 \\
\hline 'Lola' $(2 x)$ & 'Irene' $(4 x)$ & 564 & 4 & & 4 & & & & 0 \\
\hline 'Cream' $(2 x)$ & 'Carlos' $(4 x)$ & 769 & 7 & & 7 & & & & 0 \\
\hline 'Cream' $(2 x)$ & 'Dallas Red' (4x) & 995 & 4 & & 4 & & & & 0 \\
\hline 'Cream' $(2 x)$ & 'Irene' $(4 x)$ & 472 & 3 & & 3 & & & & 0 \\
\hline 'Gold' $(4 x)$ & 'Carlos' $(4 x)$ & 469 & 10 & & & 4 & & \begin{tabular}{|l|l}
$6^{2}$ \\
\end{tabular} & 60.0 \\
\hline 'Gold' $(4 x)$ & 'Dallas Red' (4x) & 668 & 5 & & & & & 5 & 100.0 \\
\hline 'Carlos' $(4 x)$ & 'Gold' (4x) & 450 & 5 & & & 5 & & & 0 \\
\hline 'Pink Caprice' $(4 x)$ & 'Denholm White' $(2 x)$ & 213 & 2 & & & & \begin{tabular}{|l|}
2 \\
\end{tabular} & & 100.0 \\
\hline 'Pink Caprice' $(4 x)$ & 'Radiation' $(4 x)$ & 273 & 4 & & & 3 & & \begin{tabular}{|l|}
1 \\
\end{tabular} & 25.0 \\
\hline 'Radiation' $(4 x)$ & 'Pink Caprice' $(4 x)$ & 193 & 4 & & & 3 & & 1 & 25.0 \\
\hline LAOP-9 $(2 x)$ & 'Carlos' $(4 x)$ & 1515 & 31 & & 31 & & & & 0 \\
\hline LAOP-9 $(2 x)$ & 'Dallas Red' (4x) & 1326 & 44 & & 44 & & & & 0 \\
\hline
\end{tabular}

${ }^{2}$ Numbers enclosed in squares are the numbers of polyploidy progeny indicating $2 n$ gamete formation.

percentages of hexaploids $(60 \%$ and $100 \%)$ appearing in these progeny indicate high percentages of $2 n$ gamete formation, more specifically, UFG formation, in 'Gold' (Table 4), because, as mentioned previously, 'Carlos' and 'Dallas Red' were shown not to produce $2 n$ gametes from controlled pollinations. When 'Carlos' was pollinated with 'Gold', all the progeny were tetraploids (Table 4). This contrasts with the ploidy level distribution in progeny of 'Gold' $\times$ 'Carlos' and indicates a lack of $2 n$ pollen formation in 'Gold' and a lack of UFG formation in 'Carlos'. 'Pink Caprice' produced pentaploids and hexaploids when pollinated with diploid 'Denholm White' or tetraploid 'Radiation', respectively, which supports the formation of UFGs in 'Pink Caprice' (25\%-100\%) (Table 4).
Transmission of UFG formation. To gain understanding of the transmission of this $2 n$ gamete formation trait, progeny of 'Gold' and 'Pink Caprice' were analyzed for ability to form UFGs. Two 'Gold' progeny were selected because of the availability of flowers for pollination. Progeny 604-1 (P604-1, $4 x$ ) was from a CP between 'Carlos' (non- $2 n$ gamete producer) and 'Gold' (UFG producer). P604-1 produced hexaploids at high frequencies in its progeny when self- or open-pollinated (Table 5). This indicates that P604-1 was able to produce $2 n$ gametes or it had inherited the trait from 'Gold'. Transmission of the trait from a paternal parent to progeny also suggests that the trait may be under nuclear gene control. Progeny GDGHOP-36 was a diploid progeny of self-pollinated 'Gold'.

Table 5. Formation of $2 n$ female gametes in the first- and/or second-generation progeny of 'Gold' and 'Pink Caprice' lantana. Pollinations were made from 2006 to 2008, and ploidy analysis done in 2008 at Wimauma, FL.

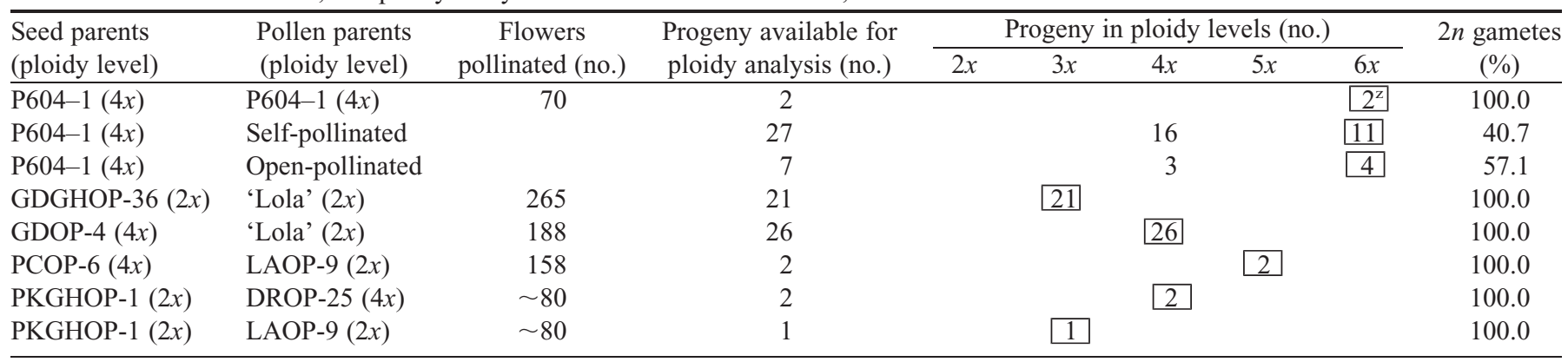

${ }^{\mathrm{Z}}$ Numbers enclosed in squares are the numbers of polyploidy progeny indicating $2 n$ gamete formation. 
When pollinated with 'Lola' ( $2 x$, non- $2 n$ female gamete producer), GDGHOP-36 produced triploids only (100\%; Table 5), indicating $2 n$ gamete formation in GDGHOP36 , another line of evidence showing the transmission of $2 n$ gamete formation from 'Gold' to its progeny.

The transmission of $2 n$ gamete formation from 'Pink Caprice' to its progeny was examined using PCOP-6 (4x), a progeny of 'Pink Caprice' and PKGHOP-1 $(2 x)$, a second-generation progeny of 'Pink Caprice'. PCOP-6 produced $2 n$ female gametes, as shown by the occurrence of $5 x$ progeny when pollinated with LAOP-9 $(2 x)$ (Table 5$)$. PKGHOP-1 produced $4 x$ progeny when pollinated with DROP-25, a $4 x$ OP progeny of 'Dallas Red' (Table 5), indicating that PKGHOP-1 also carried the UFG formation trait from 'Pink Caprice'.

EFFECT OF $2 N$ GAMETE FORMATION ON SEED PRODUCTION BY TRIPLOIDS. Three $3 x$ breeding lines with or without $2 n$ gamete formation background were pollinated with one common cultivar 'Lola' (2x) (Table 6). Breeding lines 605-35 and 624-1 were from 'Carlos' and 'Dallas Red', respectively, and both did not carry the UFG trait. Their seed set ranged from 0 to $1 \%$. Breeding line GDOP-4 carried the UFG formation trait from 'Gold', and its seed set was $25 \%$.

\section{Discussion}

In the $2 n$-gamete-producing cultivars, the frequency of $2 n$ gamete formation varied widely, from $5.5 \%$ to $100.0 \%$ in 'Gold' and from $11.3 \%$ to $100.0 \%$ in 'Pink Caprice' (Table 1). Similar variation seems common in other plants and has been noted among individuals, flowers of an individual, and even within different anthers of a single flower (McCoy, 1982; Veilleux et al., 1982). It has been documented that the frequency of $2 n$ gamete formation in a plant may be influenced by genotype, environment, and their interactions. Temperatures, in particular, seem to have a strong impact on the production of $2 n$ gametes (McHale, 1983). Additional important factors noted in previous studies are variable degrees of penetrance and expressivity in the genes responsible for $2 n$ gamete formation (Mok and Peloquin, 1975; Watanabe and Peloquin, 1989). In this study, except for the variable frequencies, a cultivar's $2 n$ gamete formation activity remained quite consistent under different pollination schemes (OP, SP, or CP) and under different growing conditions (shade house and greenhouse): $2 n$-gamete producers always produced some $2 n$ gametes, and non- $2 n$-gamete producers did not.

Two basic processes have been described as the mechanisms for $2 n$ gamete (pollen and egg) formation in plants: first division restitution (FDR) and second division restitution (SDR) (see review by Bretagnolle and Thompson, 1995). In FDR, the first meiotic division occurs abnormally, with homologous chromosomes not pairing during prophase I and/ or not separating to opposite poles during anaphase I. In SDR, the second meiotic division occurs abnormally, with sister chromatids not separating to opposite poles during anaphase II. It has been shown that univalents and multivalents (during prophase I) and laggards (during anaphase I) are very common in lantana polyploids (Natarajan and Ahuja, 1957; Spies and Stirton, 1982). It is expected, therefore, that $2 n$ gametes formed through SDR would be aneuploids and contain various numbers of chromosomes. The progeny of 'Gold' and 'Pink Caprice' resulting from UFGs were pentaploids or hexaploids (Tables 3 and 4). Similarly, the progeny of 'Red Cap' $(3 x)$ were tetraploids or pentaploids, and the progeny of 'Purple Prince' (5x) were hexaploids (Khoshoo and Mahal, 1967). These results suggest that the UFGs leading to the production of these higher level polyploids should contain all the chromosome complements in the respective seed parents. This would be possible only through FDR, and the meiotic abnormalities occurred before or during the first division and all the unpaired (univalents) as well as paired homologous chromosomes (bivalents, trivalents, and quadrivalents) did not separate during anaphase I. To confirm or refute the above inference, we are developing simple sequence repeat markers that can be used for segregation analysis and for assessing the levels of heterozygosity in the progeny of $2 n$-gamete-producing cultivars. This will help determine the stage of nonreduction.

In the present study, diploids produced triploids and tetraploids, and tetraploids produced pentaploids and hexaploids through UFG formation and fertilization with $n$ male gametes (Tables 3 and 4). In a previous study, a triploid produced tetraploid and pentaploid progeny, and a pentaploid produced hexaploid progeny through the same process (Khoshoo and Mahal, 1967). As summarized in Fig. 1, these results suggests that all the observed polyploid levels in L. camara (triploids to hexaploids) can be evolved through UFG formation and fertilization with normal $n$ male gametes, and that there exist two or more pathways for polyploidization at each of the ploidy levels in L. camara. It is conceivable that the existence of multiple polyploidization pathways could lead to an increased diversity (or complexity) in L. camara.

One interesting phenomenon in L. camara has been the lack of correlation between meiotic irregularities during microsporegenesis and seed set in naturalized polyploids. In spite of high frequencies of meiotic irregularities in pollen mother cells, lantana polyploids produced abundant viable seeds (Natarajan and Ahuja, 1957; Raghavan and Arora, 1960). Khoshoo and Mahal (1967) suggested that apoximis was responsible for the seed set in these highly male-sterile polyploids, but Spies and Stirton (1982) could not find definitive evidence for apomixis in embryo sac examination. The finding that lantana can produce UFGs may provide an explanation for such a lack of correlation in polyploids. Without undergoing the normal meiotic first division during megasporegenesis, polyploids, even though showing high levels of meiotic abnormalities during microsporegenesis in their pollen mother cells, could still produce megaspores and egg cells that contain all the chromosome complements, which would be viable and able to be fertilized and produce viable seeds.

Sterile cultivars are needed to control the invasive potential L. camara has shown in southern United States and other 


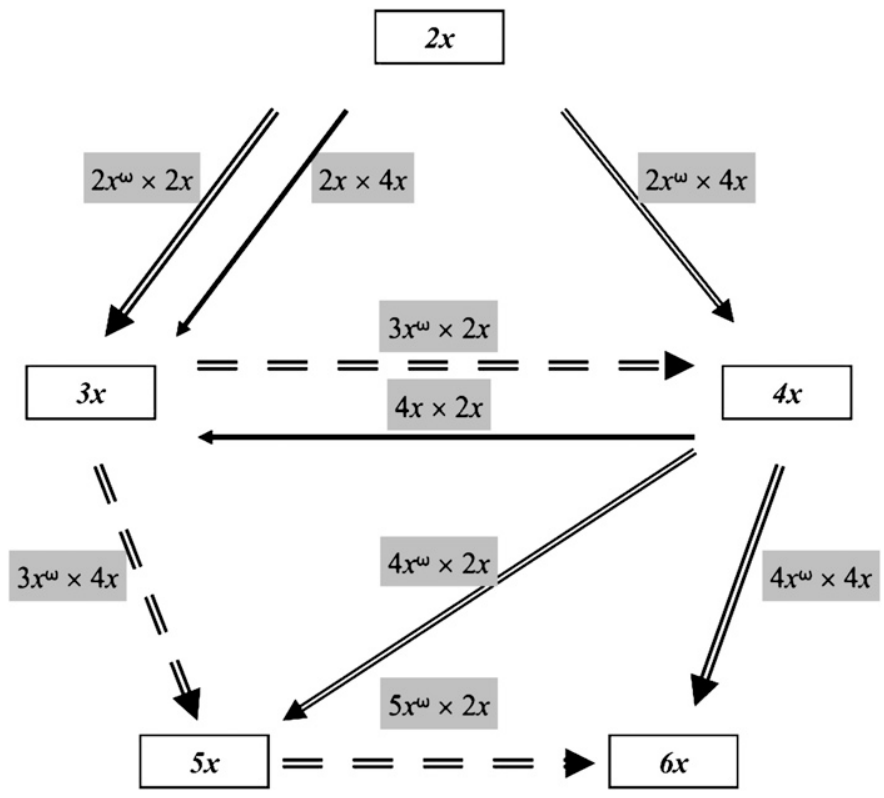

Fig. 1. Observed pathways for polyploid formation in Lantana camara. Solid double lines represent polyploidization pathways involving $2 n$ female gamete and observed in this study; dashed double lines are polyploidization pathways involving $2 n$ female gamete formation and observed by Khoshoo and Mahal (1967); and solid single lines are observed pathways not involving $2 n$ female gamete formation. Symbol $\omega$ indicates parents producing $2 n$ female gametes.

countries. Compared with diploids and tetraploids, triploid lantana plants show the lowest pollen stainability and seed set (Czarnecki et al., 2008). Production of triploids has been proposed as a main genetic approach to develop new sterile lantana cultivars. Results from the present study suggest the possibility of producing triploids through crosses between diploids and tetraploids, and through crosses between diploids as well (Fig. 1). More importantly, the results indicate a strong need to screen and select breeding parents carefully and to avoid using UFG-producing plants in crosses intended for high sterility. If triploids have inherited the ability to form UFGs from seed or pollen parents, these triploids will be likely to produce viable UFGs $(3 x)$, which can be fertilized with pollen from surrounding diploids or tetraploids and produce viable seeds, which will defy the purpose of producing such triploids. None of the diploids ('Cream', 'Denholm White', and 'Lola') in this study showed tendency to produce UFGs, but four of the seven tetraploids ('Gold', 'Pink Caprice', 'Radiation', and UPL) showed such tendency. To find more tetraploids that do not produce UFGs and can be used as breeding parents, screening of more commercial cultivars or germplasm and progeny through ploidy analysis will be required.

\section{Conclusion}

The above results showed the occurrence of $2 n$ female gametes and revealed characteristics of this phenomenon in lantana. Frequencies of UFG formation varied among cultivars and breeding lines. It was common in 'Gold', 'Pink Caprice', 'Radiation', and UPL, but it did not occur in 'Carlos', 'Dallas Red', and 'Irene'. The UFG-forming trait can be transmitted from one generation to the next, and it seems to be under nuclear gene control. This characteristic should facilitate hybridization between different ploidy levels, and thus transference of morphological or physiological traits from a diploid background to a higher ploidy background for cultivar development. Conversely, the trait could increase triploids' seed set, thus becoming a barrier to producing new sterile, triploid Lantana cultivars.

\section{Literature Cited}

Bretagnolle, F. and J.D. Thompson. 1995. Tansley Review No. 78. Gametes with the somatic chromosome number: Mechanisms of their formation and role in the evolution of autopolyploid plants. New Phytol. 129:1-22.

Czarnecki, II, D.M., Z. Deng, and D.G. Clark. 2008. Assessment of ploidy levels, pollen viability, and seed production of Lantana camara cultivars and breeding lines. HortScience 43:1195-1196. (Abstr.).

Florida Exotic Pest Plant Council. 2007. 2007 List of invasive plant species. 16 Apr. 2008. <http://fleppc.org/07list.htm>.

Hermsen, J.G. 1984. Mechanisms and genetic implications of $2 n$-gamete formation. Iowa State J. Res. 58:421-434.

Howard, R. 1969. A check list of cultivar names used in the genus Lantana. Arnoldia 29:73-109.

Khoshoo, T.N. and C. Mahal. 1967. Versatile reproduction in Lantana camara. Current Sci. Bangalore 36:201-203.

McCoy, T.J. 1982. Inheritance of $2 n$ pollen formation in diploid alfalfa (Medicago sativa L.). Can. J. Genet. Cytol. 24:315-323.

McHale, N.A. 1983. Environmental induction of high frequency $2 n$ pollen formation in diploid Solanum. Can. J. Genet. Cytol. 25:609615.

Mok, D.W.S. and S.J. Peloquin. 1975. The inheritance of three mechanisms of diplandroid ( $2 n$ pollen) formation in diploid potatoes. Heredity 35:295-302.

Natarajan, A.T. and M.R. Ahuja. 1957. Cytotaxonomical studies in the genus Lantana. J. Indian Bot. Soc. 36:35-45.

Raghavan, R.S. and C.M. Arora. 1960. Morphological and cytological studies in the genus Lantana L. Bul. Bot. Survey India 2:299-303.

Rambuda, T.D. and S.D. Johnson. 2004. Breeding systems of invasive alien plants in South Africa: Does Baker's rule apply? Divers. Distrib. 10:409-416.

Ramsey, J. and D.W. Schemske. 1998. Pathways, mechanisms, and rates of polyploidy formation in flowering plants. Annu. Rev. Ecol. Syst. 29:467-501.

Ranney, T.G. 2004. Population control: Developing non-invasive nursery crops. Combined Proc. Intl. Plant Propagators' Soc. 54: 604-607.

Sanders, R.W. 1987. Identity of Lantana depressa and L. ovatifolia (Verbenaceae) of Florida and the Bahamas. Syst. Bot. 12:44-60.

Sanders, R.W. 2001. The genera of Verbenaceae in the southeastern United States. Harv. Pap. Bot. 5:303-358.

Sharma, G.P., A.S. Raghunbanshi, and J.S. Singh. 2005. Lantana invasion: An overview. Weed Biol. Manage. 5:157-165.

Sinha, S. and A. Sharma. 1984. Lantana camara L.: A review. Feddes Repert. 95:621-633.

Spies, J.J. and C.H. Stirton. 1982. Embryo sac development in some South African cultivars of Lantana camara. Bothalia 14:113-117.

Stelly, D.M. and S.J. Peloquin. 1986. Formation of $2 n$ megagametophytes in diploid tuber-bearing solanums. Amer. J. Bot. 73:13511363.

Veilleux, R.E., N.A. McHale, and F.I. Lauer. 1982. Unreduced gametes in diploid Solanum phureja Juz. and Buk. Theor. Appl. Genet. 59:95-100.

Watanabe, K. and S.J. Peloquin. 1989. Occurrence of $2 n$ pollen and $p s$ gene frequencies in cultivated groups and their related wild species in tuber-bearing Solanums. Theor. Appl. Genet. 78:329-336.

Wirth, F.F., K.J. Davis, and S.B. Wilson. 2004. Florida nursery sales and economic impacts of 14 potentially invasive ornamental plant species. J. Environ. Hort. 22:12-16. 